\title{
Vorwort zur 10, Auflage
}

Mit der ro. Auflage ist das Buch in seiner inneren und äußeren Gestaltung grundlegend geändert worden.

Schon die letzten Auflagen (insbesondere die neunte) dienten in zunehmendem Maße der Erreichung eines doppelten Zieles: Einmal an Hand von Fällen mit Lösungen zu zeigen, wie man in logischem Aufbau und klarer Disposition aus einem komplizierten Lebensvorgang den juristischen Kern herausschält, zum anderen aber die in innerem Zusammenhang mit den jeweiligen Lösungen stehenden Gebiete des Strafgesetzbuches und seiner Nebengesetze in „Vorbemerkungen" und "Nachträgen" lehrbuchartig zu erfassen und, auf eine einfache Formel gebracht, in übersichtlicher und einprägsamer Darstellung zu erörtern. Diese Zielsetzung hat mit der vorliegenden Neuauflage ihre Verwirklichung gefunden. Um diesen Abschluß in der inneren Entwicklung des Buches von einer reinen Fallsammlung mit Lösungen zu einem auf praktischen Fällen aufgebauten Lehrbuch auch äußerlich in Erscheinung treten zu lassen, wurde der Buchtitel durch den Zusatz „Ein induktives Strafrechtslehrbuch" erweitert (siehe hierzu die in der Juristischen Rundschau 1950, Heft I erschienene Kritik zur 9. Auflage).

Im einzelnen sei zu der inneren Neugestaltung des Buches folgendes bemerkt: Den „Fällen“, die in der Neuauflage wesentlich geändert, erweitert und teilweise erneuert wurden, liegt zum Teil Examensmaterial aus der zweiten juristischen Prüfung des ehemaligen Badischen Justizministeriums zugrunde. In der Hauptsache aber sind sie das Ergebnis einer in jahrelanger Tätigkeit als Staatsanwalt und Strafrichter erworbenen praktischen Erfahrung. Bei ihrer Zusammenstellung war das Bestreben maßgebend, nicht theoretisch interessante Tatbestände zu konstruieren, sondern wirklichkeitstreue Lebensvorgänge, soweit sie sich auf dem Gebiet des Strafrechts bewegen, zur Darstellung zu bringen. Nach wie vor bilden diese, in einem besonderen Heft zusammengeiaßten und in einer Schlaufe des hinteren Einbanddeckels untergebrachten 24 Fälle das Fundament des Buches. Daneben aber wurden - in Abweichung von den früheren Auflagen - die lehrbuchartigen 
Ausführungen in den "Nachträgen" durch zahlreiche kleinere Fälle mit Lösungen bzw. durch kurze Beispiele bereichert.

Die den Lösungen vorangestellten "Systematischen Vorbemerkung en" (Seite 39-59), die in den bisherigen Auflagen fehlten, bilden den Ausgangspunkt für die Lösungen. Sie enthalten eine Aufgliederung der Grundelemente der strafbaren Handlung und befassen sich besonders ausführlich mit dem Schuldproblem und der neuen Irrtumslehre.

Die „Lösungen" wurden auf Grund der pädagogischen Erfahrungen, die ich in langjährigen Vorbereitungskursen zum Assessorexamen gesammelt habe, insofern umgestaltet, als die rein theoretischen Erörterungen von den eigentlichen Lösungen vollkommen getrennt und ausschließlich in den „Vorbemerkungen" und „Nachträgen" untergebracht wurden. Die "Lösungen" sowohl wie die theoretischen Ausführungen sind aufgebaut in der Hauptsache auf der Judikatur des Reichsgerich ts und der in der amtlichen Sammlung des Bundesgerich tsh ofs (bis einschließlich Band 5, Heft 4/5) niedergelegten Rechtsprechung. Daneben wurden auch - in beschränktem Maße - oberlandesgerichtliche Entscheidungen verwertet. Der ursprüngliche Plan, einer Anregung aus Universitätskreisen folgend auch das Schrifttum zu berücksichtigen (siehe die in der ,Zeitschrift für die gesamte Strafrechtswissenschaft" Band 65 , Heft 4/1953 veröffentlichte Kritik zur 9. Auflage), konnte im Hinblick auf die durch Preisgestaltungsfragen bedingte Notwendigkeit, den Umfang des Buches in bestimmten Grenzen zu halten, in dieser Auflage noch nicht verwirklicht werden. Unbearbeitet bleiben vorläufig die 5 ersten Abschnitte des Zweiten Teils des Strafgesetzbuches $(\$ \S 80-\log a)$, da diese Tatbestände sich für eine Zusammenfassung in „Fälle“ weniger eignen und überdies die veröffentlichte höchstrichterliche Judikatur auf diesen Gebieten bis jetzt nur einen geringen Umfang hat. Ebenso mußten, durch den Charakter dieses Lehrbuchs bedingt, verschiedene, den ersten Abschnitt des Allgemeinen Teils $(\$ \S 14-42)$ betreffende Gesetzesstellen außerhalb einer Erörterụng bleiben.

Was schließlich die äußere Neugestaltung des Buches betrifft, erschien im Hinblick auf den nunmehr lehrbuchartigen inneren Aufbau eine Teilung in zwei getrennte Bände (,Erster Teil“, „Zweiter Teil") zweckmäßig. Der vorliegende „Erste Teil", in dem die ersten Io Fälle bearbeitet sind, befaßt sich in der Hauptsache mit den Allgemeinen Lehren des Strafrechts ( $\left.\S I_{-}-79\right)$ und den für die tägliche Strafrechtspraxis wichtigsten Tatbeständen. Der „Zweite Teil" des Buches, der Ende des Jahres erscheinen wird, 
enthält die Lösungen zu den weiteren i4 Fällen, die die sämtlichen, im „Ersten Teil"“ noch nicht behandelten Gebiete aus dem Allgemeinen und Besonderen Teil des Strafgesetzbuches, sowie die wichtigsten strafrechtlichen Nebengesetze zum Gegenstande haben.

Das Inhaltsverzeichnis wurde zum Zwecke einer raschen Orientierung ergänzt durch ein Gesetzesregister. Ein ausführliches alphabetisches Sachregister wird dem „Zweiten Teil“ des Buches beigegeben werden.

So möge die seit langem angestrebte und nunmehr mit der ro. Auflage verwirklichte Kombination einer Fallsammlung mit einem Lehrbuch dem juristischen Nachwuchs noch wirksamer als bisher die Vorbereitungsarbeit zu den juristischen Prüfungen mit ihren gesteigerten Anforderungen erleichtern. Dabei verkenne ich nicht, daß eine solche, vollkommen neuartige Form eines Lehrbuchs keinen geschlossenen Gesamtüberblick gewähren kann und insofern weniger für den Anfänger, als vor allem für den „fortgeschrittenen“ Studenten und für den Referendar in Frage kommt. Darüber hinaus wird das Buch aber auch dem Strafrechtspraktiker die Möglichkeit geben, sich über schwierige allgemeine Probleme des Strafrechts und die für die tägliche Praxis wichtigsten Straftatbestände in ihrer Auslegung nach der neuesten Rechtsprechung des Bundesgerichtshofs zu orientieren.

Heidelberg, im August 1954

Dr. Petters

\section{Vorwort zur 12. Auflage}

Wegen der inneren und äußeren Gestaltung sowie der Zweckbestimmung dieses induktiven Lehrbuchs wird auf das oben zum Abdruck gebrachte Vorwort zur ı. Auflage Bezug genommen. Auch bei dem vorliegenden, die ersten to Fälle betreffenden Teil des Gesamtwerkes (bezeichnet mit „Erster Teil") wurde zunächst die seit Erscheinen der II. Auflage neu hinzugekommene höchstrichterliche Rechtsprechung bis einschließlich Band 15 der amtlichen Sammlung in Strafsachen verarbeitet. Dabei wurde auch das neuere Schrifttum in größerem Umfange als bisher berücksichtigt. 